\title{
Autonomic dysfunction in chronic liver disease
}

James Frith

Julia L Newton

NIHR Biomedical Research Centre in Ageing, Institute for Ageing and Health, Newcastle University, Newcastle UK
Correspondence: Julia L Newton Professor of Ageing and Medicine, NIHR Biomedical Research Centre in Ageing - Liver theme, Institute for Ageing and Health Newcastle University, Newcastle, NE2 4HH, UK Tel +44 I9I 2824I 28

Email julia.newton@nuth.nhs.uk
This article was published in the following Dove Press journal:

Hepatic Medicine: Evidence and Research

22 August 201I

Number of times this article has been viewed

Abstract: It is becoming increasingly clear that quality of life (QOL) is impaired in those with chronic liver disease (CLD). One of the most important contributors to impaired QOL is the symptomatic burden which can range from slight to debilitating. Autonomic dysfunction accounts for a significant proportion of these symptoms, which can be common, non-specific and challenging to treat. Investigating the autonomic nervous system can be straight forward and can assist the clinician to diagnose and treat specific symptoms. Evidence-based treatment options for autonomic symptoms, specifically in CLD, can be lacking and must be extrapolated from other studies and expert opinion. For those with severely impaired quality of life, liver transplantation may offer an improvement; however, more research is needed to confirm this.

Keywords: quality of life, treatment, fatigue, angiotensin II

\section{Introduction}

With the ever-expanding population, improvements in treatment, and the subsequent improvement in survival, the chronic liver disease (CLD) population is expanding. While advancing prognosis may be considered beneficial for patients a longer life does not always equate to an improved quality of life. ${ }^{1,2}$ One of the major contributors to a poor quality of life in CLD is autonomic dysfunction $(\mathrm{AD})^{3-5}$ and while the evidence base for the treatment of CLD continues to expand, the evidence for symptom control and palliation is being left behind.

Autonomic dysfunction is an impairment of the normal functioning of the autonomic nervous system that adversly affects health. It can be primary or secondsary, acute or chronic, and transient or progressive. This review will focus on chronic, secondary AD as a result of CLD. The prevalence of AD in CLD is as high as 67\% in some populations ${ }^{6}$ and cannot therefore be ignored. Furthermore, the importance of $\mathrm{AD}$ is highlighted by its associated increase in mortality. ${ }^{6}$ With this in mind it is essential that clinicians who care for people with CLD recognize when AD may be present, and which investigations and treatments may be of benefit.

\section{Search strategy and selection criteria}

Publications from 1950 to date were searched using OvidSP. Search terms used included 'autonomic nervous system' and 'liver disease'. Excluding non-English papers 350 papers were identified. The resulting reference list of this paper is composed of articles reviewed and selected by both authors with additional relevant papers identified through reference lists. Where there is a lack of evidence in the literature regarding a specific 
point general principles and expert opinion are applied: these comments are explicit when included.

\section{The autonomic nervous system (ANS)}

The autonomic nervous system is complex and as yet not fully understood. The essential points will be discussed here to aid the clinical understanding of AD. The sympathetic (SNS) and parasympathetic nervous systems (PNS) form the two arms of the ANS through which viscera, smooth muscle and secretory glands are controlled involuntarily.

Sympathetic preganglionic neurons release acetylcholine, whereas postganglionic neurons transmit noradrenaline (with the exception of sweat glands which are cholinergic, and the adrenal medulla which directly synapses with preganglionic neurons). The physiological responses of sympathetic nervous stimulation are increased heart rate (HR) and blood pressure (BP); dilatation of the bronchi; vasodilatation to skeletal muscle; vasoconstriction to the gastrointestinal tract; decreased gastrointestinal motility; increased sweating; smooth muscle sphincter contraction and pupillary mydriasis.

The neurotransmitter of the parasympathetic nervous system is acetylcholine, through which, under stimulation, the following clinical responses occur: decreased HR and contractility; vasodilation; bronchial constriction; increased gastrointestinal motility; relaxation of smooth muscle sphincters and pupilary miosis.

\section{Pathogenesis}

With the pathophysiology of CLD being so multifarious, the interaction it has with the ANS is difficult to fully appreciate. CLD results in complex changes in electrolyte homeostasis, altered regulation and response to vasocontrictors and dilators, changes to the arterio-venous circulation and other immunological and physiological changes which place additional stresses on autonomic function.

Liver disease results in a decreased response to vasoconstrictors which may be caused by increased concentration of vasodilators such as nitric oxide. An increase in portal blood pressure, even mild, can lead to an up-regulation of nitric oxide synthetase. ${ }^{7}$ An increase in circulating vasodilators, a diseased liver and a portosystemic circulation bypassing hepatic metabolism will contribute to increased systemic vasodilatation. Circulating vasodilators will activate the rennin-angiotensin-aldosterone system (RAAS) and increase plasma levels of the vasoconstrictor angiotensin II. In support of this theory is the increased plasma concentration of angiotensin II in patients with chronic liver disease (CLD). ${ }^{8}$
Angiotensin II may interact with the parasympathic control of heart rate variability (HRV). Infusion of angiotensin II causes a decrease in HRV and a reduction in vagal discharges to the heart; if the vagus nerve is severed there is no change in HRV when it is infused. Administration of an angiotensin converting enzyme inhibitor improves the HRV confirming that angiotensin II plays a role in $\mathrm{AD} .{ }^{9}$ In addition, administration of antioxidants seems to reverse blunted BP responses suggesting that oxidative stress may also play a role in $\mathrm{AD} .{ }^{10}$

$\mathrm{AD}$ is common in diabetic patients, and in those who suffer iatrogenic hypoglycemia there is a hypoglycemia-associated autonomic failure (HAAF). ${ }^{11}$ This is a result of decreased response of adrenaline and the SNS to hypoglycemia. It may be possible that the hypoglycemia induced by severe hepatic dysfunction may bring about HAAF. The resultant AD creates hypoglycemic unawareness, and therefore a vicious circle of worsening $\mathrm{AD}{ }^{12}$

$\mathrm{AD}$ in advanced liver disease is associated with decreased baroreceptor sensitivity to hypotension, leading to impaired BP and HR responses. ${ }^{13}$

\section{Prevalence of AD in CLD}

Figure 1 describes the prevalence of AD in specific CLD populations.

\section{Presentation of AD in CLD}

Disease-specific features of AD are described in Table 1.

The clinical picture of a patient with chronic liver disease presenting with $\mathrm{AD}$ is similar for $\mathrm{AD}$ of any cause. However, the presentation can be subtle, easily missed and with the aforementioned cardiovascular changes the presentation

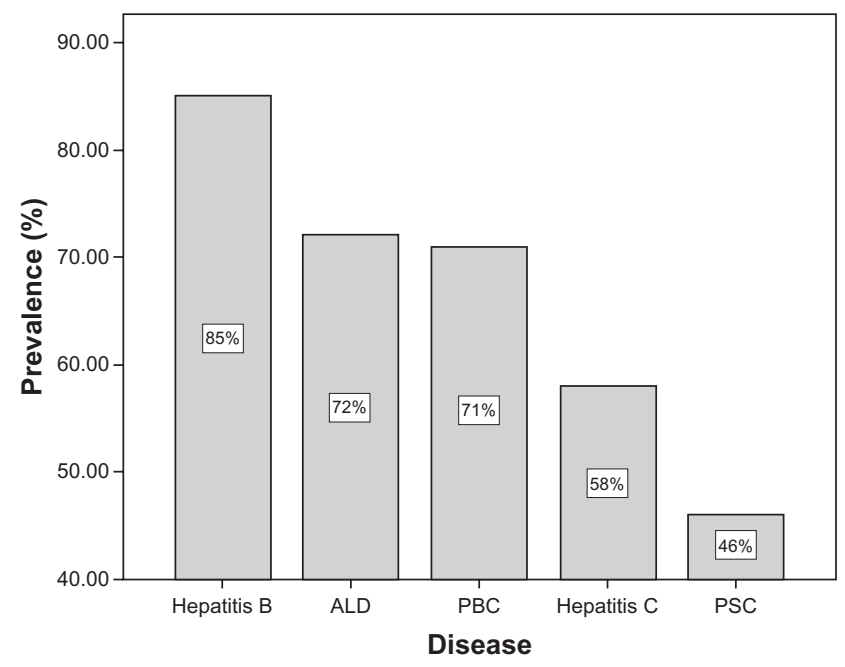

Figure I Prevalence of autonomic dysfunction in specific chronic liver diseases. ${ }^{48-52}$ Abbreviations: ALD, alcoholic liver disease; PBC, primary biliary cirrhosis; PSC, primary sclerosing cholangitis. 
Table I Reported clinical features of autonomic dysfunction in chronic liver disease

Cognitive decline

Postural dizziness

Falls

Fatigue

Urinary incontinence

Early satiety/nausea

Sexual dysfunction

can be more complex and difficult to distinguish from other sequelae of CLD, such as hypovolemia.

\section{Investigating the ANS}

The simplest, clinical test for AD is to test for orthostatic hypotension $(\mathrm{OH})$ by measuring lying and standing BP. A diagnosis of $\mathrm{OH}$ is made if there is a systolic drop in BP of $\geq 20 \mathrm{~mm} \mathrm{Hg}$ or a diastolic drop of $\geq 10 \mathrm{~mm} \mathrm{Hg}$ within 3 minutes. ${ }^{14} \mathrm{~A}$ diagnosis of positional orthostatic tachycardia syndrome (POTS) can be made if there is a symptomatic increase in $\mathrm{HR}$ of $\geq 30$ beats per minute (bpm) using the same postural challenge, in the absence of a BP drop. ${ }^{15}$ In patients with CLD, before a diagnosis of $\mathrm{OH}$ is made, hypervolemia should be excluded. Typically hypovolemia will cause an isolated systolic drop with no diastolic drop in pressure, however this is unreliable.

Another simple clinical test is a resting electrocardiogram. A normal resting HR would lie between 60 and $80 \mathrm{bpm}$ : levels above this could indicate parasympathetic dysfunction. However anemia, hyperthyroidism, sepsis, hypovolemia, arrhythmias and pheochromocytomas can also cause resting tachycardias. The presence of rate-limiting medication can also mask parasympathetic dysautonomia.

Symptom assessment tools are also available and may be more appropriate than physiologic testing where specialist equipment such as beat-to-beat BP and HR monitoring are not available. The Orthostatic Grading Scale (OGS) may be the most simple to use as it consists of only 10 items which are scored by patients according to the severity of postural symptoms. ${ }^{16}$ It is widely used but has not been fully validated in the CLD population. A further disadvantage is that it only assesses symptoms of $\mathrm{OH}$. Conversely, the Composite Autonomic Symptom Scale (COMPASS) is much more detailed and thorough in its assessment of systemic AD but at the price of being cumbersome, lengthy and more difficult to complete. ${ }^{17}$

Other tests of autonomic function are available (see Table 2), but these are specialized and not practicable in every clinic. However an understanding of them is of value in order to understand specialist investigation and research. Measuring autonomic function is unreliable in those patients who are on beta-blockers, as normal sympathetic responses will be blunted.

\section{Treating AD in CLD}

As there is currently no cure for dysautonomia, the principles of treatment are symptomatic. It is important to investigate and exclude reversible causes of symptoms masquerading as AD. For example a urinary tract infection can cause urinary symptoms, fatigue and tachycardia. There are numerous factors which complicate the management of dysautonomia in the patient with CLD. Patients may be on diuretics, betablockers, antidepressants or sedatives, all of which affect the autonomic nervous system. Lactulose and diuretics as discussed previously can contribute to incontinence or sexual dysfunction, and a risk/benefit analysis may need to be undertaken with the patient. An additional treatment which challenges the ANS is ascitic paracentesis. The reduction in intra-abdominal pressure can result in profound splanchnic vasodilation resulting in a prominent activation of the SNS leading to, at worst, circulatory collapse. This is avoided by infusion of intravenous albumin to maintain a normovolemic circulation.

Evidence is lacking for the treatment of dysautonomia in CLD and much must be extrapolated from the evidence relating to $\mathrm{AD}$ in general. In general conservative measures are recommended as first line treatment but when these fail specialist advice should be sought.

\section{Specific dysautonomic symptoms Orthostatic hypotension}

One of the commonest consequences of AD in CLD is orthostatic hypotension $(\mathrm{OH}) .{ }^{18}$ The patient typically complains of dizziness or lightheadedness on standing from a sitting or lying position. Other symptoms include blurring of vision, discomfort of head, neck or shoulder (the so called 'coat hanger' headache), fatigue, and in severe cases, syncope.

Patients with $\mathrm{OH}$ should try to maintain their intravascular volume by drinking at least 2-2.5 liters of fluid per day. This may however, complicate CLD, especially those who are fluid overloaded but who are unable to maintain their fluid in the intravascular compartment. Compression stockings help to redistribute extravascular fluid, and also prevent peripheral venous pooling on assuming an upright posture. Large meals should be avoided to minimize postprandial hypotension, and all patients should adopt a slow, gradual staged movement on rising into standing position. Pharmacological options are 
Table 2 Tests of autonomic function, and classification of severity ${ }^{53}$

\begin{tabular}{|c|c|}
\hline & Method \\
\hline Active standing (parasympathetic) & Wearing an ECG monitor the subject rests supine to achieve stable baseline HR levels. \\
\hline$\geq 1.04$ normal & The subject then stands and the ratio is calculated of the longest RRI (around the thirtieth beat) \\
\hline I.0I-1.03 borderline & to the shortest RRI (around the fifteenth beat) \\
\hline \multicolumn{2}{|l|}{$<1.0$ abnormal } \\
\hline Deep breathing (parasympathetic) & With ECG monitoring in a sitting position the patient breathes deeply and evenly at 6 breaths \\
\hline$>9$ bpm normal (age $>60)$ & per minute. The maximum and minimum HR during each cycle is noted for 3 consective cycles. \\
\hline$\geq 15$ bpm normal $($ age $<60)$ & The mean difference between maximum and minimum $H R$ is \\
\hline II-14 bpm borderline & calculated \\
\hline \multicolumn{2}{|l|}{$\leq 10 \mathrm{bpm}$ abnormal } \\
\hline Valsalva (parasympathetic) & After resting, the patient expires for 15 seconds against a closed glottis (pressure of $40 \mathrm{mmHg}$ ). \\
\hline$\geq 1.1$ I normal (age > 60) & The ratio of the longest RRI just after the valsalva, and the short RRI during the strain, \\
\hline I.I I-1.2 borderline & is calculated \\
\hline \multicolumn{2}{|l|}{$\geq 1.21$ normal $($ age $<60)$} \\
\hline Valsalva (sympathetic) & The same method as for valsalva (parasympathetic) but BP is recorded \\
\hline \multicolumn{2}{|c|}{$\begin{array}{l}\text { In normal patients there will be a BP overshoot } \\
\text { shortly after releasing the strain }\end{array}$} \\
\hline Isometric exercise (sympathetic) & Using a dynamometer, hand grip is maintained at $30 \%$ of maximum grip for 5 minutes. Diastolic \\
\hline$\geq 16 \mathrm{mmHg}$ normal & pressure is measured before exercise and just before release, the difference is calculated \\
\hline \multicolumn{2}{|l|}{ II-I5 mmHg borderline } \\
\hline \multicolumn{2}{|l|}{$\leq 10 \mathrm{mmHg}$ abnormal } \\
\hline $\begin{array}{l}\text { Cold pressor (sympathetic) } \\
\geq 15 \mathrm{mmHg} \text { normal }\end{array}$ & $\begin{array}{l}\text { One hand is held in iced water for I minute. Diastolic BP is measured, before and after, the increase } \\
\text { is calculated }\end{array}$ \\
\hline \multicolumn{2}{|l|}{ I $-14 \mathrm{mmHg}$ borderline } \\
\hline \multicolumn{2}{|l|}{$\leq 10 \mathrm{mmHg}$ abnormal } \\
\hline \multicolumn{2}{|l|}{ Dysautonomia may be classified as: } \\
\hline \multicolumn{2}{|l|}{ - None: all tests normal or borderline } \\
\hline \multicolumn{2}{|c|}{ - Early: one abnormal HR test or two borderline } \\
\hline \multicolumn{2}{|c|}{ - Definite: two or more abnormal HR tests } \\
\hline \multicolumn{2}{|c|}{ - Severe: two or more HR rate tests abnormal, plus one borderline or abnormal BP test } \\
\hline - Atypical: any other combination o & ests \\
\hline
\end{tabular}

Abbreviations: HR, heart rate; BP, blood pressure; RRI, R-R interval; ECG, electrocardiogram; bpm, beats per minute.

available for those few who do not respond to conservative measures. Fludrocortisone can improve $\mathrm{OH}$ by causing sodium and water retention, but caution is required in CLD where secondary hyperaldosteronism may already be present. Midodrine, which is used off-license in the United Kingdom, improves $\mathrm{OH}$ through agonist action on $\alpha 1$-adrenoreceptors. Extreme caution should be taken when used in patients with CLD, as it can worsen liver function. Other agents which have been used are beta-blockers, clonidine, pyridostigmine, erythropoietin and selective serotonin re-uptake inhibitors. Generally, the treatment of $\mathrm{OH}$ in the presence of portal hypertension is challenging.

\section{Fatigue}

Fatigue is a common complaint of patients with CLD and ranges from slight impairment of daily living to severe debilitation. Liver function tests (LFTs) are often performed in the patient who presents with 'tiredness all the time' and fatigue may therefore be a presenting symptom of CLD. It can develop at any time in patients with existing liver disease, but it does not correlate with disease severity. ${ }^{19,20}$ The severity of fatigue experienced by patients correlates with increasingly severe autonomic dysfunction, and must therefore be a major contributing factor in the etiology of this symptom. ${ }^{21}$ Other contributing factors may include metabolic disturbance, anemia, altered sleep patterns and medication (benzodiazepines, antidepressants, beta-blockers).

In addition to fatigue, autonomic dysfunction can also reduce exercise tolerance. In patients with dysautonomia secondary to diabetes a blunted response of HR, BP, adrenaline and noradrenaline is seen. ${ }^{22}$ The same response can be anticipated in those with AD in CLD.

Fatigue is often multi-factorial and difficult to manage. In CLD-associated fatigue, patients should be encouraged to adopt a steady state of activity, and avoid intense bouts of activity when energy is present. In resistant cases of primary biliary cirrhosis (PBC) where fatigue is associated with excessive daytime sleepiness modafinil has been used successfully. ${ }^{23}$ It is relatively contraindicated in hypertensive patients, and BP should be monitored while on treatment. Starting dose 
is $50-100 \mathrm{mg}$ and titrated accordingly. Side effects include headaches, sleep disturbance and hypertension. In those who suffer from insomnia a sedative may improve sleep thereby improving daytime wakefulness. In those who suffer broken sleep or display risk factors for sleep apnea a referral for sleep studies should be considered. In patients on beta-blockers a risk/benefit analysis should be undertaken on an individual basis, as beta-blockers can cause profound fatigue.

\section{Urinary symptoms}

Bladder dysfunction is a feature of AD; $14 \%$ of pre-transplant patients use incontinence pads. ${ }^{24}$ Although there is very little data concerning bladder dysfunction in CLD we know from other AD associated diseases that it may also manifest as urinary frequency, hesitation or retention; the consequences of which include urinary tract infections, renal failure and indignity. ${ }^{25}$ Incontinence is exacerbated by prescribing diuretics.

\section{Gastrointestinal problems}

In patients with CLD there is both delayed gastric emptying of liquids and solids and a prolonged transit time from mouth to cecum, ${ }^{26,27}$ symptoms include early satiety, nausea, vomiting, weight loss and epigastric pain. The decrease in small bowel motility resulting from AD is particularly important as it can cause bacterial overgrowth, with increased risk of encephalopathy and peritonitis. Delayed gastric emptying is associated with post-prandial hypoglycemia, and may therefore worsen AD. ${ }^{28}$ Changes in gastrointestinal motility alongside disturbances of sphincter control can cause diarrhea, constipation and incontinence. Incontinence is exaggerated by prescribing laxatives.

Current treatments for delayed gastric emptying are, on the whole, disappointing. Medical management includes erythromycin which improves motility but does not improve symptoms, and domperidone which seems to be more effective at symptom control, in diabetic patients with gastroparesis. ${ }^{29}$ Surgical options as yet are limited as trials have been retrospective and uncontrolled, but gastrostomy, botulinum neurotoxin and implantable electrodes to stimulate motility have shown promise..$^{29-31}$

Gastrointestinal mobility disorders may be initially managed with diet, followed by medications such as laxatives or bulking agents, and constipating agents with planned enemas. For severe, resistant cases with significant impact on daily living surgical options exist. For incontinence the anal sphincter can be repaired or substituted, but with disappointing long term success. ${ }^{32}$ For incontinence and constipation colectomy with or without stoma formation may improve symptoms. ${ }^{33}$

The treatment of urinary problems caused by dysautonomia is limited; urodynamic studies will help direct appropriate management. First line treatment for urinary frequency includes bladder retraining (increasing time between voiding) or regular toileting (micturating every 2-4 hours). Pharmacological treatments include antimuscarinics which can worsen $\mathrm{AD}$ and alpha-blockers which can cause profound $\mathrm{OH}$. For severely hypotonic bladders intermittent catheterization may reduce incontinence, infections and renal failure. ${ }^{34}$

\section{Sexual function}

LFTs may be performed when investigating a patient who presents with sexual dysfunction, and may identify liver disease. Sexual problems may arise either from fatigue, altered body image, AD or from alterations in circulating sex hormones. Females show higher levels of dysfunction in end stage liver disease. ${ }^{35}$ Diuretics, beta-blockers and antidepressants can all exacerbate sexual dysfunction.

It is difficult to distinguish between $\mathrm{AD}$, medication adverse effects and psychological disturbance as the cause of sexual dysfunction. Contributing medications should be reviewed. Despite lower levels of testosterone and increased levels of estrogen, supplementing testosterone is not effective at improving erectile dysfunction. ${ }^{36}$ Unsurprisingly, sexual dysfunction does improve in alcoholic men who abstain. ${ }^{37}$ The incidence of erectile dysfunction more than doubles in men who undergo transjugular intrahepatic portosystemic stenting. ${ }^{38}$

\section{Liver transplantation (LT) and autonomic function}

The majority (87\%) of patients who undergo liver transplantation suffer from AD. ${ }^{39}$ The degree of dysautonomia improves after liver transplantation in $63 \%$ of patients at 6-7 months; however in a very small number it gets worse despite successful grafting, ${ }^{39}$ and in patients whose allograft function is impaired, dysautonomia persists. ${ }^{40}$ Although the aforementioned studies had relatively short follow-up studies of spontaneous baroreflex sensitivity have shown improvements which remain at 18 months. ${ }^{41}$

The effects of LT on sexual and urinary dysfunction are much less impressive, with no improvements in symptoms. This confirms the complex nature of these problems with medication and psychological factors perhaps having a greater effect than $\mathrm{AD} .^{24,35}$ 


\section{Prognosis}

The association between liver disease severity and the incidence of $\mathrm{AD}$ is equivocal, with conflicting results. ${ }^{6,42-44}$ Fouryear mortality is $30 \%$ in people with CLD and dysautonomia compared to $6 \%$ in those with CLD and no dysautonomia. ${ }^{45}$ $\mathrm{AD}$ is an independent risk factor for mortality in both compensated and decompensated cirrhotic patients. ${ }^{6}$ In one study which followed patients awaiting LT for 10 months, 6 patients died. Each had AD, leading the authors to conclude that consideration should be given to early LT in those with AD. ${ }^{6}$ In addition to increased mortality in those with cardiovascular abnormalities, patients with $\mathrm{PBC}$ who suffer from fatigue have an increased mortality. ${ }^{46}$

The aforementioned sequelae of AD add significantly to poorer quality of life in patients already burdened with chronic disease. Recent studies are also beginning to define additional potential consequences of $\mathrm{AD}$ in those with liver disease such as cognitive impairment. ${ }^{47}$ As dysautonomia is increasingly common in older age we should expect to see more consequences of $\mathrm{AD}$ as the liver disease population ages. Falls, $\mathrm{OH}$, incontinence and cognitive impairment are all more common with age and will pose significant problems in this population.

Although the increase in mortality associated with AD is significant, hepatologists should not solely focus on survival, but address symptomatic burden and quality of life. Challenging, vague symptoms can often be improved through active listening and empathy, improving patients' expectations and satisfaction. If patient care is to be improved, outcome measures which enable patients to represent the treatment effects which make a real difference to their lives must be incorporated into routine clinical practice. $^{5}$

\section{Conclusion}

Although greater understanding of the pathogenesis of $\mathrm{AD}$ in CLD is required, its effects on patients are becoming increasingly recognized. The symptomatic burden of patients with CLD and dysautonomia is high, but may be overlooked in favor of laboratory markers of disease severity. Many of the symptoms, such as fatigue and sexual dysfunction, pose difficulties for clinicians, as they may be considered low priority, ubiquitous and unmodifiable. Simple measures such as reviewing medications (beta-blockers, diuretics, and antidepressants) may lead to improvements in the patients' symptoms and quality of life. Recognizing AD will help to identify those patients who are at increased risk of death, and may contribute to the consideration for LT.

\section{Disclosure}

The authors report no conflicts of interest in this work.

\section{References}

1. Marchesini G, Bianchi G, Amodio P, et al. Factors associated with poor health-related quality of life of patients with cirrhosis. Gastroenterology. 2001;120(1):170-178.

2. David K, Kowdley KV, Unalp A, et al. Quality of life in adults with nonalcoholic fatty liver disease: baseline data from the nonalcoholic steatohepatitis clinical research network. Hepatology. 2009;49(6):1904-1912.

3. Newton J, Pairman J, Wilton K, Jones DE, Day C. Fatigue and autonomic dysfunction in non-alcoholic fatty liver disease. Clin Auton Res. 2009;19(6):319-326.

4. Kramer L, Hofer H, Bauer E, et al. Relative impact of fatigue and subclinical cognitive brain dysfunction on health-related quality of life in chronic hepatitis C infection. AIDS. 2005;19 Suppl 3:S85-S92.

5. Jones DEJ, Sutcliffe K, Pairman J, Wilton K, Newton JL. An integrated care pathway improves quality of life in primary biliary cirrhosis. QJM. 2008;101(7):535-543.

6. Fleckenstein JF, Frank S, Thuluvath PJ. Presence of autonomic neuropathy is a poor prognostic indicator in patients with advanced liver disease. Hepatology. 1996;23(3):471-475.

7. Abraldes JG, Iwakiri Y, Loureiro-Silva M, Haq O, Sessa WC, Groszmann RJ. Mild increases in portal pressure upregulate vascular endothelial growth factor and endothelial nitric oxide synthase in the intestinal microcirculatory bed, leading to a hyperdynamic state. Am J Gastrointest Liver Physiol. 2006;290(5):G980-G987.

8. Arroyo V, Planas R, Gaya J, et al. Sympathetic nervous activity, reninangiotensin system and renal excretion of prostaglandin E2 in cirrhosis. Relationship to functional renal failure and sodium and water excretion. Eur J Clin Invest. 1983;13(3):271-278.

9. Dillon JF, Nolan J, Thomas H, et al. The correction of autonomic dysfunction in cirrhosis by captopril. J Hepatol. 1997;26:331-335.

10. Ferlitsch A, Pleiner J, Mittermayer F, et al. Vasoconstrictor hyporeactivity can be reversed by antioxidants in patients with advanced alcoholic cirrhosis of the liver and ascites. Crit Care Med. 2005;33(9): 2028-2033.

11. Cryer PE. Hypoglycemia in diabetes: pathophysiological mechanisms and diurnal variation. Prog Brain Res. 2006;153:361-365.

12. Cryer PE. Diverse causes of hypoglycemia-associated autonomic failure in diabetes. $N$ Engl J Med. 2004;350(22):2272-2279.

13. Lhuillier F, Dalmas ED, Gratadour PM, et al. Spontaneous baroreflex cardiac sensitivity in end-stage liver disease: effect of liver transplantation. Eur J Anaesthesiol. 2006;23(5):426-432.

14. Anonymous. Consensus statement on the definition of orthostatic hypotension, pure autonomic failure, and multiple system atrophy. The Consensus Committee of the American Autonomic Society and the American Academy of Neurology. Neurology. 1996;46(5): 1470.

15. Low PA, Opfer-Gehrking TL, Textor SC, et al. Postural tachycardia syndrome (POTS). Neurology. 1995;45(4 Suppl 5):S19-S25.

16. Schrezenmaier C, Gehrking JA, Hines SM, Low PA, Benrud-Larson LM, Sandroni P. Evaluation of orthostatic hypotension: relationship of a new self-report instrument to laboratory-based measures. Mayo Clin Proc. 2005;80(3):330-334.

17. Suarez GA, Opfer-Gehrking TL, Offord KP, Atkinson EJ, O’Brien PC, Low PA. The Autonomic Symptom Profile: A new instrument to assess autonomic symptoms. Neurology. 1999;52(3):523-528.

18. Chaudhry V, Corse AM, O'Brian R, Cornblath DR, Klein AS, Thuluvath PJ. Autonomic and peripheral (sensorimotor) neuropathy in chronic liver disease: a clinical and electrophysiologic study. Hepatology. 1999;29(6):1698-1703.

19. Newton JL, Jones DE, Henderson E, et al. Fatigue in non-alcoholic fatty liver disease (NAFLD) is significant and associates with inactivity and excessive daytime sleepiness but not with liver disease severity or insulin resistance. Gut. 2008;57(6):807-813. 
20. Cauch-Dudek K, Abbey S, Stewart DE, Heathcote EJ. Fatigue in primary biliary cirrhosis. Gut. 1998;43(5):705-710.

21. Newton JL, Davidson A, Kerr S, et al. Autonomic dysfunction in primary biliary cirrhosis correlates with fatigue severity. Eur J Gastroenterol Hepatol. 2007;19(2):125-132.

22. Bottini P, Tantucci C, Scionti L, et al. Cardiovascular response to exercise in diabetic patients: influence of autonomic neuropathy of different severity. Diabetologia. 1995;38(2):244-250.

23. Jones DE, Newton JL. An open study of modafinil for the treatment of daytime somnolence and fatigue in primary biliary cirrhosis. Aliment Pharmacol Ther. 2007;25(4):471-476.

24. Gomez-Lobo V, Burgansky A, Kim-Schluger L, Berkowitz R. Gynecologic symptoms and sexual function before and after liver transplantation. J Reprod Med. 2006;51(6):457-462.

25. Haensch CA, Jorg J. Autonomic dysfunction in multiple sclerosis. J Neurol. 2006;253 Suppl 1:I3-I19.

26. Galati JS, Holdeman KP, Dalrymple GV, Harrison KA, Quigley EM. Delayed gastric emptying of both the liquid and solid components of a meal in chronic liver disease. Am J Gastroenterol. 1994;89(5):708-711.

27. Galati JS, Holdeman KP, Bottjen PL, Quigley EM. Gastric emptying and orocecal transit in portal hypertension and end-stage chronic liver disease. Liver Transpl Surg. 1997;3(1):34-38.

28. Ohlsson B, Melander O, Thorsson O, Olsson R, Ekberg O, Sundkvist G Oesophageal dysmotility, delayed gastric emptying and autonomic neuropathy correlate to disturbed glucose homeostasis. Diabetologia. 2006;49(9):2010-2014.

29. Bloomgarden ZT. Diabetic neuropathy. Diabetes Care. 2008;31(3): 616-621.

30. Lacy BE, Crowell MD, Schettler-Duncan A, Mathis C, Pasricha PJ The treatment of diabetic gastroparesis with botulinum toxin injection of the pylorus. Diabetes Care. 2004;27(10):2341-2347.

31. Abell T, McCallum R, Hocking M, et al. Gastric electrical stimulation for medically refractory gastroparesis. Gastroenterology. 2003;125(2): 421-428.

32. Lehur PA, Glemain P, Bruley des Varannes S, Buzelin JM, Leborgne J. Outcome of patients with an implanted artificial anal sphincter for severe faecal incontinence. A single institution report. Int $J$ Colorectal Dis. 1998;13(2):88-92.

33. Kamm MA, Hawley PR, Lennard-Jones JE. Outcome of colectomy for severe idiopathic constipation. Gut. 1988;29(7):969-973.

34. Shamliyan TA, Kane RL, Wyman J, Wilt TJ. Systematic review: randomized, controlled trials of nonsurgical treatments for urinary incontinence in women. Ann Intern Med. 2008;148(6):459-473.

35. Sorrell JH, Brown JR. Sexual functioning in patients with endstage liver disease before and after transplantation. Liver Transpl. 2006;12(10):1473-1477.

36. Gluud C, Wantzin P, Eriksen J. No effect of oral testosterone treatment on sexual dysfunction in alcoholic cirrhotic men. Gastroenterology. 1988;95(6):1582-1587.
37. Van Thiel DH, Gavaler JS, Sanghvi A. Recovery of sexual function in abstinent alcoholic men. Gastroenterology. 1983;84(4):677-682.

38. Nolte W, Schindler CG, Figulla HR, et al. Increase of serum estradiol in cirrhotic men treated by transjugular intrahepatic portosystemic stent shunt. J Hepatol. 2001;34(6):818-824.

39. Carey EJ, Gautam M, Ingall T, Douglas DD. The effect of liver transplantation on autonomic dysfunction in patients with end-stage liver disease. Liver Transpl. 2008;14(2):235-239.

40. McDougall AJ, Davies L, McCaughan GW. Autonomic and peripheral neuropathy in endstage liver disease and following liver transplantation. Muscle Nerve. 2003;28(5):595-600.

41. Lhuillier F, Dalmas ED, Gratadour PM, et al. Spontaneous baroreflex cardiac sensitivity in end-stage liver disease: effect of liver transplantation. Eur J Anaesthesiol. 2006;23(5):426-432.

42. Hendrickse MT, Triger DR. Peripheral and cardiovascular autonomic impairment in chronic liver disease: prevalence and relation to hepatic function. $J$ Hepatol. 1992;16(1-2):177-183.

43. Oliver MI, Miralles R, Rubies-Prat J, et al. Autonomic dysfunction in patients with non-alcoholic chronic liver disease. J Hepatol. 1997;26(6): $1242-1248$.

44. Trevisani F, Sica G, Bernardi M. Autonomic neuropathy in advanced liver disease. Hepatology. 1996;24(6):1549.

45. Hendrickse MT, Thuluvath PJ, Triger DR. Natural history of autonomic neuropathy in chronic liver disease. [see comment]. Lancet 1992;339(8807):1462-1464.

46. Jones DE, Bhala N, Burt J, Goldblatt J, Prince M, Newton JL. Four year follow up of fatigue in a geographically defined primary biliary cirrhosis patient cohort. Gut. 2006;55(4):536-541.

47. Newton J, Hollingsworth K, Taylor R, et al. Cognitive impairment in primary biliary cirrhosis: Symptom impact and potential etiology. Hepatology. 2008;48(2):541-549.

48. Szalay F, Marton A, Keresztes K, Hermanyi ZS, Kempler P. Neuropathy as an extrahepatic manifestation of chronic liver diseases. Scand $J$ Gastroenterol Suppl. 1998;228:130-132.

49. Newton JL, Gibson GJ, Tomlinson M, Wilton K, Jones D. Fatigue in primary biliary cirrhosis is associated with excessive daytime somnolence. Hepatology. 2006;44(1):91-98.

50. Newton JL, Hudson M, Tachtatzis P, et al. Population prevalence and symptom associations of autonomic dysfunction in primary biliary cirrhosis. Hepatology. 2007;45(6):1496-1505.

51. Lindgren S, Lilja B, Verbaan H, Sundkvist G. Alcohol abuse exaggerates autonomic dysfunction in chronic liver disease. Scand J Gastroenterol. 1996;31(11):1120-1124.

52. Monforte R, Estruch R, Valls-Sole J, Nicolas J, Villalta J, UrbanoMarquez A. Autonomic and peripheral neuropathies in patients with chronic alcoholism. A dose-related toxic effect of alcohol. Arch Neurol. 1995;52(1): 45-51.

53. Ewing DJ, Clarke BF. Autonomic neuropathy: its diagnosis and prognosis. Clin Endocrinol Metab. 1986;15(4):855-888.
Hepatic Medicine: Evidence and Research

\section{Publish your work in this journal}

Hepatic Medicine: Evidence and Research is an international, peerreviewed, open access journal covering all aspects of adult and pediatric hepatology in the clinic and laboratory including the following topics: Pathology, pathophysiology of hepatic disease; Investigation and treatment of hepatic disease; Pharmacology of drugs used for

\section{Dovepress}

the treatment of hepatic disease. Issues of patient safety and quality of care will also be considered. The manuscript management system is completely online and includes a very quick and fair peer-review system, which is all easy to use. Visit http://www.dovepress.com/ testimonials.php to read real quotes from published authors. 